\title{
IS VERTICAL TRANSMISSION OF CORONAVIRUS (COVID-19) FROM MOTHER TO BABY POSSIBLE?
}

\section{ABSTRACT}

The coronavirus (COVID-19) pandemic has serious health implications. The potential risk to pregnant women and neonates must be explored. Controversy exists regarding whether Severe Acute Respiratory Syndrome Coronavirus 2 (SARS-CoV-2) can be transmitted vertically via antenatal or intrapartum transmission from an infected mother to her child. Emerging case reports and cohort studies suggest that vertical transmission is likely. However, the data is limited and contradictory, making it difficult to draw definitive conclusions. The continued collection and analysis of data on pregnant women and neonates is necessary.

\section{Key words}

COVID-19, Pregnancy, vertical transmission
TRIYA CHAKRAVORTY BA (OXON)1, MARIA MEMTSA MRCOG2, REHAN KHAN MD FRCOG 3

1 Clinical Medical School, University of Oxford, UK

2 University College London, UK

3 Royal London Hospital, London, UK

Triya.chakravorty@queens.ox.ac.uk

Article Information

ePub

08.04.2020

Final v2 01.06 .2020

See peer review at physicianjnl.net

Cite as: Chakravorty T, Memtsa M, Khan R. (2020) Vertical transmission of coronavirus (COVID-19) from mother to baby possible? The Physician 6(1) DOI: 10.38192/1.6.1.3

\section{Introduction}

With the number of people infected with coronavirus (COVID-19) globally having reached over one million, it is clear that this pandemic has serious health implications1. Severe acute respiratory syndrome coronavirus 2 (SARS-CoV2), which leads to COVID-19, is a highly infectious virus with multiple possible routes of transmission. Currently, controversy exists regarding whether SARS-CoV-2 can be transmitted vertically from an infected mother to her unborn child. Understanding the impact of the virus on pregnant women and the foetus is necessary for generating guidelines for obstetric, and subsequent neonatal, management regarding COVID-19 infection. This is an important issue to consider, given that changes to the immune system mean that pregnant women, although not necessarily more susceptible to viral illness, may have more severe symptoms, especially in the last trimester2. In the case of COVID-19, more severe complications include viral pneumonitis, respiratory failure and acute respiratory distress syndrome. These are seen in high risk groups such as older patients, immunosuppressed and those with certain longterm health conditions3. Similar symptoms could occur in pregnant women, making identification and treatment important.

Evidence regarding the possibility of vertical transmission

Emerging case reports and cohort studies from Wuhan, China the epicentre of the pandemic, 
the physician

suggest that vertical transmission is likely4. However, results are inconsistent, and studies involve small sample sizes, which is unavoidable given the recent emergence of COVID-19. Chen et als conducted a retrospective analysis of nine pregnant women with COVID-19 pneumonia admitted to Zhongnan Hospital, Wuhan in January 2020. In this cohort, they found no evidence of vertical transmission, which was assessed by testing for the presence of SARSCoV-2 in amniotic fluid, cord blood and neonatal throat swab samples. However, other case studies suggest that vertical transmission is possible. For example, Zeng et al 6 conducted a cohort study involving 33 neonates born to mothers with COVID-19 in Wuhan Children's Hospital. They found that three neonates (9\%) tested positive for COVID-19 and showed symptoms of the viral illness. In this cohort, the neonates were born via caesarean section under strict infection control measures. Based on this, the authors concluded that the three neonates likely contracted the disease in utero. However, it must be noted that the diagnostic test for COVID-19 was not carried out until the second day of life, which raises the possibility that infection occurred after birth cannot be ruled out.

Further evidence for the possibility of vertical transmission is provided by Dong et al7, who wrote to the Journal of the American Medical Association (JAMA) about a neonate born to a mother with COVID-19 in Renmin Hospital, Wuhan in February 2020. This neonate had elevated SARS-CoV-2 IgM and IgG antibody levels and abnormal cytokine test results two hours after birth. The elevated levels of SARS-CoV-2 IgM antibody present suggests that the neonate was infected in-utero, as IgM antibodies do not cross the placenta, and therefore likely represent a neonatal immune response to in-utero infection8. The mother's diagnosis of COVID-19 took place 23 days before delivery, making this the potential time frame for vertical transmission.

Although infection at delivery cannot be ruled out, the precautionary measures taken at the time of birth (including delivery via caesarean section in a negative-pressure isolation room, mother wearing an $\mathrm{N} 95$ mask, no motherneonate contact and immediate quarantine of the neonate in the neonatal intensive care unit) decrease this. Unlike the study conducted by Chen et al, in this case study, the mother's vaginal secretions were tested and found to be negative for SARS-CoV-2, which further supports the possibility that the neonate developed COVID-19 via vertical transmission. However, it was a single case study, and therefore can only be considered a preliminary observation, which warrants further examination of maternal and neonatal samples.

It must be noted that in the case studies mentioned, the women contracted COVID-19 in their third trimester, which means that the possibility of vertical transmission during the first or second trimester remains unexplored. This is an important consideration, since this time period is immunologically significant, as demonstrated by rubella infections that can affect more than $50 \%$ of foetuses if maternal infection occurs in the first trimester, whereas the incidence rate halves in cases that maternal infection occurs by the end of the second trimesterg. It is also imperative to consider standardisation of the diagnostic tests used in the neonatal period.

Impact of COVID-19 on pregnant women and neonates

The impact of COVID-19 infection on pregnant women and neonates, and whether this differs from the impact of the virus on other population groups is still under consideration. Currently, the proportion of pregnancies affected by COVID-19 is not known2. For example, during the $2009 \mathrm{H}_{1} \mathrm{~N}_{1}$ influenza pandemic, pregnant women were at an increased risk of complications and were over four times more likely to be admitted to hospital compared to the general populationio. Furthermore, in a case study of 12 pregnant women infected with SARS-CoV in 2003, the mortality rate was $25 \% 11$, compared to the average global mortality of $10 \% 12$. However, in a cohort of nine pregnant women with COVID-19, the clinical characteristics of COVID-19-related pneumonia were similar to those reported for non-pregnant adults5. Once again, the lack of large sample sizes makes it difficult to 


\section{Wrom the physician}

confidently draw any conclusions. Currently, pregnant women are not classed as extremely vulnerable in the Public Health England guidelines13.

There is currently no evidence to suggest that COVID-19 infection is related to an increased risk of early pregnancy loss2. Based on case reports from early pregnancy studies with Severe Acute Respiratory Syndrome (SARS-CoV) and Middle East Respiratory Syndrome (MERS-CoV), maternal viral infection was not associated with an increased risk of miscarriage or second trimester loss14.

At present, there is limited data regarding the clinical course of COVID-19 in neonates. However, the current consensus is that in children, severe disease from COVID-19 is rare 15. In the largest review to date of children with COVID-19, only 112 (5\%) of 2143 children had severe disease (defined as hypoxia) and 13 $(0.6 \%)$ of children developed respiratory or multiorgan failure or acute respiratory distress syndrome16. In a case study conducted by Zeng et $a l_{6}$, the three infected neonates had fever and pneumonia, which are symptoms typically associated with the virus. For two out of three them, the clinical course of disease was mild. The third neonate required treatment for nonCOVID-19-related conditions including bacterial sepsis and prematurity. However, it is not known whether these conditions were impacted by COVID-19 in any way.

\section{Conclusion}

Vertical transmission may indeed occur in COVID-19 infection. Due to the limited data available, it is not possible to draw definitive conclusions. However, considering the significance of this ongoing pandemic, it is imperative to continue to collect and analyse data on pregnant women and neonates. For the time being, screening of pregnant women, close monitoring of at-risk neonates and strict perinatal infection control measures should be carried out in all cases.

References:
1 Henley, J. More than a million confirmed cases of Covid-19 globally, <https://www.theguardian.com/world/2020/ap r/02/top-european-teaching-hospitals-runningout-of-coronavirus-drugs > (2020).

2 RCOG. Guidance for healthcare professionals on coronavirus (COVID-19) infection in pregnancy, published by the RCOG, Royal College of Midwives, Royal College of Paediatrics and Child Health, Public Health England and Health Protection Scotland., <https://www.rcog.org.uk/globalassets/docume nts/guidelines/2020-04-03-coronavirus-covid19-infection-in-pregnancy.pdf> (2020).

3 Guan, W.-j. et al. Clinical Characteristics of Coronavirus Disease 2019 in China. New England Journal of Medicine, doi:10.1056/NEJMoa2002032 (2020).

$4 \quad$ Zhu, N. et al. A Novel Coronavirus from Patients with Pneumonia in China, 2019. N Engl J Med

382 ,

727-733, doi:10.1056/NEJMoa2001017 (2020).

5 Chen, H. et al. Clinical characteristics and intrauterine vertical transmission potential of COVID-19 infection in nine pregnant women: a retrospective review of medical records. The Lancet 395, 809-815, doi:10.1016/S01406736(20)30360-3 (2020).

6 Zeng, L. et al. Neonatal Early-Onset Infection With SARS-CoV-2 in 33 Neonates Born to Mothers With COVID-19 in Wuhan, China. JAMA Pediatrics, doi:10.1001/jamapediatrics.2020.0878 (2020).

7 Dong, L. et al. Possible Vertical Transmission of SARS-CoV-2 From an Infected Mother to Her Newborn. JAMA, doi:10.1001/jama.2020.4621 (2020).

8 Palmeira, P., Quinello, C., Silveira-Lessa, A. L., Zago, C. A. \& Carneiro-Sampaio, M. IgG placental transfer in healthy and pathological pregnancies. Clin Dev Immunol 2012, 985646, doi:10.1155/2012/985646 (2012).

9 Bouthry, E. et al. Rubella and pregnancy: diagnosis, management and outcomes. Prenat Diagn 34, 1246-1253, doi:10.1002/pd.4467 (2014).

10 Gottfredsson, M. [The Spanish flu in Iceland 1918. Lessons in medicine and history]. Laeknabladid 94, 737-745 (2008).

11 Wong, S. F. et al. Pregnancy and perinatal outcomes of women with severe acute 
respiratory syndrome. Am J Obstet Gynecol 191, 292-297, doi:10.1016/j.ajog.2003.11.019 (2004). 12 Who. (World Health Organization, 2003).

13 Public Health England - Guidance on shielding and protecting people defined on medical grounds as extremely vulnerable from COVID-19,

<https://www.gov.uk/government/publications /guidance-on-shielding-and-protectingextremely-vulnerable-persons-from-covid19/guidance-on-shielding-and-protectingextremely-vulnerable-persons-from-covid-19> (2020).

14 Zhang, J. P., Wang, Y. H., Chen, L. N., Zhang, R. \& Xie, Y. F. [Clinical analysis of pregnancy in second and third trimesters complicated severe acute respiratory syndrome]. Zhonghua Fu Chan Ke Za Zhi 38, 516-520 (2003). 15 Sinha, I. P. et al. COVID-19 infection in children. The Lancet Respiratory Medicine, doi:10.1016/S2213-2600(20)30152-1.

16 Dong, Y. et al. Epidemiological Characteristics of 2143 Pediatric Patients With 2019 Coronavirus Disease in China. Pediatrics, e20200702, doi:10.1542/peds.2020-0702 (2020). 